\title{
TITLE:
}

\section{Hydrogen-deuterium exchange on plasma-exposed W and SS surface}

\section{$\operatorname{AUTHOR}(\mathrm{S}):$}

Takagi, Ikuji; Nomura, Shinji; Minamimoto, Toshihiro; Akiyoshi, Masafumi; Kobayashi, Taishi; Sasaki, Takayuki

\section{CITATION:}

Takagi, Ikuji ...[et al]. Hydrogen-deuterium exchange on plasma-exposed W and SS surface. Journal of Nuclear Materials 2015, 463: 1125-1128

\section{ISSUE DATE:}

2015-08

URL:

http://hdl.handle.net/2433/202010

\section{RIGHT:}

(c) 2014. This manuscript version is made available under the CC-BY-NC-ND 4.0 license

http://creativecommons.org/licenses/by-nc-nd/4.0/; The full-text file will be made open to the public on 14 Novembe 2016 in accordance with publisher's 'Terms and Conditions for Self-Archiving'.; この論文は出版社版でありません。引 用の際には出版社版をご確認ご利用ください。; This is not the published version. Please cite only the published version. 


\title{
Hydrogen-deuterium exchange on plasma-exposed W and SS surface
}

\author{
Ikuji Takagi*, Shinji Nomura, Toshihiro Minamimoto, Masafumi Akiyoshi, Taishi \\ Kobayashi, Takayuki Sasaki \\ Department of Nuclear Engineering, Kyoto University, Japan
}

\begin{abstract}
The desorption cross section for hydrogen isotopes adsorbed on stainless steel (SS) and tungsten (W) has been evaluated experimentally to provide basic information on tritium exchange. One side of a sample sheet was alternately exposed to $\mathrm{H}$ and $\mathrm{D}$ plasma, and deuterium density on the surface was repeatedly observed using nuclear reaction analysis (NRA) under continuous plasma exposure. From the time dependent change in the deuterium density, the desorption cross sections for SS and W were estimated to be $6.9 \pm 2.3 \times 10^{-23} \mathrm{~m}^{2}$ and $4.6 \pm 1.0 \times 10^{-23} \mathrm{~m}^{2}$, respectively. No significant differences in the cross section between $\mathrm{H}$ and D plasma were observed. Recombinative desorption was found to dominate the desorption process owing to the low incident energy of hydrogen atoms.
\end{abstract}

PACS: 34.35.+a, 25.55.-e, 52.20.Hv

PSI-21 keywords: Deuterium inventory, Hydrogen, Desorption, Stainless steel, Nuclear reaction analysis, Tungsten, Exchange

*Corresponding author address: Kyoto daigaku-Katsura, Nishikyo-ku, Kyoto 615-8540, Japan

*Corresponding author E-mail: takagi@nucleng.kyoto-u.ac.jp

Presenting author: I. Takagi

Presenting author E-mail: takagi@nucleng.kyoto-u.ac.jp 


\section{Introduction}

In DT fusion devices, tritium inventory in plasma-facing materials is an important safety issue [1]. Isotopic exchange by exposure to plasma [2-3] is effective in reducing the tritium inventory near the surface region [4]. For a better understanding of elementary processes, isotopic exchange experiments on SS using keV energy particles have been performed [5-7]. Blewer et al. [5] have shown that removal of $\mathrm{D}$ by $\mathrm{H}$ ions is described by a two-term exponential function. Bastasz et al. [7] have clearly shown an exponential decrease in the amount of adsorbed $\mathrm{D}$ atoms by bombardment of $1 \mathrm{keV} \mathrm{H}_{3}{ }^{+}$. Recently, experiments on tungsten [8-10] have shown that retained $\mathrm{D}$ near the surface is effectively reduced by exposure to $\mathrm{H}$ plasma.

In the above works, a cross section is used [5-8] for the characterization of the hydrogen exchange. This cross section represents the efficiency of hydrogen isotope removal from a specific bulk region and consists of several processes such as replacement, trapping, detrapping and diffusion. As the removed hydrogen atom is finally desorbed from the surface, a cross section for hydrogen desorption from the surface is also an important parameter. In the present work, sample plates are alternately exposed to $\mathrm{H}$ and $\mathrm{D}$ plasma, and the evolution of the deuterium surface density is observed in situ using nuclear reaction analysis (NRA). The cross section for deuterium desorption is then examined.

\section{Experimental}

Tungsten (W) and type-304 stainless steel (SS) sheets with a thickness of $0.1 \mathrm{~mm}$ provided by Nilaco Co. were used as samples. The W sheet purity was $99.95 \%$ and the main impurities were Mo (40 ppm), Fe (20 ppm), P (20 ppm), C (15 ppm), and O (10 ppm). The composition of the SS sheet was Fe (71.7\%), Cr (18.2\%), Ni(8.3\%), Mn (1.1\%), Si (0.59\%), and C (0.05\%). Table 1 shows a list of samples. All W samples and the SS5 sample were mechanically polished with abrasive paper and finished with an alumina powder of $0.05 \mu \mathrm{m}$. The other SS samples were used as received.

A typical experimental procedure was as follows. One side of a sample was exposed to weakly-ionized D plasma at room temperature, which was monitored by a thermocouple. The flux and the average energy of the deuterium atoms from the plasma were $1.0 \times 10^{19} \mathrm{~m}^{-2} \mathrm{~s}^{-1}$ 
[11] and around $1 \mathrm{eV}$ [12], respectively. During continuous plasma exposure, a beam of 1.7$\mathrm{MeV}^{3} \mathrm{He}$ from the 4MV Van de Graaff Accelerator at Kyoto University was injected into the plasma-exposed side of the sample and deuterium depth profiles were observed by converting energy spectra of protons produced by $\mathrm{D}\left({ }^{3} \mathrm{He}, \mathrm{p}\right){ }^{4} \mathrm{He}$. Details of the experimental system have been described elsewhere [13]. After the deuterium concentration reached steady state, the D plasma was switched to $\mathrm{H}$ plasma and the depth profiles were again observed. Table 1 summarizes the plasma exposure sequence.

A deuterium depth profile at steady state of SS2-1, where SS2 and 1 represent the sample and the run numbers listed in Table 1, respectively, is shown in Fig. 1. Almost all deuterium atoms are present on the surface and few deuterium atoms are present in the bulk region since the sample temperature is too low for deuterium to diffuse into deeper depths. Note that deuterium seems to be present at negative depths due to the finite depth resolution of the NRA system. The depth resolution is estimated to be $0.09 \mu \mathrm{m}$ at FWHM (full width at half maximum) using the figure. Here the deuterium density on the surface is taken as an integral value of the deuterium concentration between $-0.15 \mu \mathrm{m}$ and $0.15 \mu \mathrm{m}$. The deuterium density in Fig. 1 is 5.0 $\times 10^{19} \mathrm{~m}^{-2}$. It is much larger than the monolayer density of solid metal (approximately $2 \times 10^{19}$ $\mathrm{m}^{-2}$ ) because the actual area is larger than the apparent area owing to surface roughness [14].

\section{Analytical Model}

As the incident energy of the atoms in the present work is very low, only processes on the surface will be considered, that is, processes concerned with bulk such as diffusion, trapping, implantation, and entering into bulk from surface will not be taken into account. Fig. 2 shows possible processes of (a) sticking, (b) reflection on the surface, (c) reflection on hydrogen, (d) recombinative desorption, (e) replacement, and (f) surface recombination. Processes concerned with molecular hydrogen ions are assumed to be included in the above processes. For example, (g), desorption by a molecular ion of $\mathrm{H}_{3}{ }^{+}$can be regarded as being the same process as (d).

Surface coverage, $\theta$, which is the ratio of hydrogen density to adsorption site density, $S_{0}$, is used in the following equations. The deuterium density at steady state is taken to be $S_{0}$, which will be discussed later. The particle reflection coefficient for low-energy hydrogen atoms 
on a hydrogen-free surface is close to 0 [15], while on a hydrogen-covered surface this coefficient is very high [16]. So the particle reflection coefficient for processes (b) and (c) are assumed to be 0 and 1, respectively. In a recombinative desorption process (d), four cases of an incident and a target atom are possible, that is, H-H, H-D, D-H, and D-D. It is assumed that the cross section for each process is the same value of $\sigma_{d}$ since no significant differences in exchange behaviors between $\mathrm{H}$ and $\mathrm{D}$ have been reported $[9,17]$. For the same reason, the cross section $\sigma_{r}$ for replacement process (e) and the rate constant $k$ for surface recombination process (f) are assumed to be identical for $\mathrm{H}$ and $\mathrm{D}$. Time dependent changes in $\theta_{\mathrm{H}}$ and $\theta_{\mathrm{D}}$ are then expressed as;

$$
\begin{aligned}
& S_{0} d \theta_{\mathrm{H}} / d t=F_{\mathrm{H}}\left(1-\theta_{\mathrm{H}}-\theta_{\mathrm{D}}\right)-S_{0} \sigma_{d} \theta_{\mathrm{H}}\left(F_{\mathrm{H}}+F_{\mathrm{D}}\right)+S_{0} \sigma_{r}\left(F_{\mathrm{H}} \theta_{\mathrm{D}}-F_{\mathrm{D}} \theta_{\mathrm{H}}\right)-k\left(\theta_{\mathrm{H}}^{2}+\right. \\
& \left.\theta_{\mathrm{H}} \theta_{\mathrm{D}} / 2\right) \quad \text {, } \\
& S_{0} d \theta_{\mathrm{D}} / d t=F_{\mathrm{D}}\left(1-\theta_{\mathrm{H}}-\theta_{\mathrm{D}}\right)-S_{0} \sigma_{d} \theta_{D}\left(F_{\mathrm{H}}+F_{\mathrm{D}}\right)+S_{0} \sigma_{r}\left(F_{\mathrm{D}} \theta_{\mathrm{H}}-F_{\mathrm{H}} \theta_{\mathrm{D}}\right)-k\left(\theta_{\mathrm{D}}^{2}+\right. \\
& \left.\theta_{\mathrm{H}} \theta_{\mathrm{D}} / 2\right) \quad \text {, }
\end{aligned}
$$

where $F$ is the incident atom flux.

In a preliminary experiment, the sample was exposed to D plasma to reach steady state. The plasma was subsequently turned off, and the deuterium density was observed under vacuum conditions. Fig. 3 shows that $\theta_{\mathrm{D}}$ did not significantly decrease during a 6 ks period for SS2-2 and W1-2, nor during $43 \mathrm{ks}$ for SS1-2. In the case of vacuum exposure, $F_{\mathrm{H}}=F_{\mathrm{D}}=0$ and, in this case, $\theta_{\mathrm{H}}=0$, then Eq. (1b) can be analytically solved by

$$
\theta_{\mathrm{D}}=\left(1 / \theta_{\mathrm{D} 0}-k t / S_{0}\right)^{-1} \quad,
$$

where $\theta_{\mathrm{D} 0}$ is the initial value of $\theta_{\mathrm{D}} \cdot \theta_{\mathrm{D} 0}$ is close to unity, as mentioned previously, and $k$ is $4 \times 10^{13} \mathrm{~m}^{-2} \mathrm{~s}^{-1}$ at $298 \mathrm{~K}$ for $\mathrm{W}$ [11]. When a time, $t$, of $20 \mathrm{ks}$ is given, $k t / S_{0}$ is $8 \times 10^{-3}$, which is much smaller than $1 / \theta_{\mathrm{D} 0}$, so $\theta_{\mathrm{D}}$ does not decrease within this time. The surface recombination process is not considered and $k$ is taken as 0 hereafter.

In the experiment, the $\mathrm{H}_{2}$ feed gas was switched to $\mathrm{D}_{2}$ after $\theta_{\mathrm{D}}$ reached steady state, and 
vice versa. As $\theta_{\mathrm{H}}$ cannot be directly observed by the NRA, the steady state of $\theta_{\mathrm{H}}$ is estimated from a time dependent change in $\theta_{\mathrm{D}}$, assuming that behaviors of hydrogen and deuterium are the same. The initial conditions are expressed by $\theta_{j}(0)=0$ and $\theta_{i}(0)=\theta_{i 0}$ to indicate switching from a hydrogen isotope $i$ to another hydrogen isotope $j$. Then Eqs. (1a) and (1b) can be simplified as follows,

$$
\begin{aligned}
& \theta_{i}=\theta_{i 0} \exp \left(-F_{j} \sigma t\right), \\
& \theta_{j}=\theta_{i 0}\left[1-\exp \left(-F_{j} \sigma t\right)\right], \\
& \theta_{i 0}=\left(1+S_{0} \sigma_{d}\right)^{-1},
\end{aligned},
$$

where $\sigma \equiv \sigma_{d}+\sigma_{r}$ and $\sigma$ represents the apparent cross section for hydrogen desorption. Note that $F_{i}$ is constant and $F_{j}=0$ at $t<0$, and that $F_{i}=0$ and $F_{j}$ is constant at $t \geq 0$. For example, Eq. (1b) yields Eq. (5) as $\theta_{\mathrm{D} 0}=\left(1+S_{0} \sigma_{d}\right)^{-1}$ under conditions of $F_{\mathrm{D}}$ being constant, $F_{\mathrm{H}}=$ 0 and $d \theta_{\mathrm{D}} / d t=0$. Eq. (1b) yields Eq. (3) as $\theta_{\mathrm{D}}=\theta_{\mathrm{D} 0} \exp \left[-F_{\mathrm{H}}\left(\sigma_{d}+\sigma_{r}\right) t\right]$ under conditions of $F_{\mathrm{H}}$ being constant and $F_{\mathrm{D}}=0$ at $t \geq 0$. Then Eq. (4) as $\theta_{\mathrm{H}}=\theta_{\mathrm{D} 0}\{1-$ $\left.\exp \left[-F_{\mathrm{H}}\left(\sigma_{d}+\sigma_{r}\right) t\right]\right\}$ is derived by substituting the above solutions into Eq. (1a), or more easily, Eq. (4) is directly obtained from Eq. (3) and a relation of $\theta_{\mathrm{H}}+\theta_{\mathrm{D}}=\theta_{\mathrm{D} 0}$ which is confirmed by adding each side of Eqs. (1a) and (1b).

\section{Results and Discussion}

When D plasma was switched to $\mathrm{H}$ plasma at $t=0$, the deuterium density in SS3-2 monotonically decreased, as shown by circles in Fig. 4. The error bar represents a statistical error. The data are analyzed using Eq. (3) assuming that $\theta_{\mathrm{D}}$ at $t<0$ is equal to $\theta_{\mathrm{D} 0}$. The result is represented by the solid line at $t \geq 0$ and the desorption cross section $\sigma$ is estimated to be $4.9 \times 10^{-23} \mathrm{~m}^{2}$, where an offset of $0.8 \times 10^{19} \mathrm{~m}^{-2}$ in the deuterium density is taken into account. The offset can be attributed to deuterium located in the bulk near the surface since most deuterium atoms entering the bulk region cannot migrate to deeper depths owing to a low diffusion coefficient. When the $\mathrm{H}$ plasma was switched to D plasma, the deuterium density increased as shown by squares in Fig. 4, which can be well reproduced by calculation using Eq. 
(4) and $\sigma=4.4 \times 10^{-23} \mathrm{~m}^{2}$. The estimated cross section for each SS sample is listed in Table 1 . The average values of $\sigma$ for the $\mathrm{H}$ and D plasma are $7.2 \pm 1.6 \times 10^{-23} \mathrm{~m}^{2}$ and $6.5 \pm 2.9 \times 10^{-23}$ $\mathrm{m}^{2}$, respectively. There are no significant differences in $\sigma$ between the $\mathrm{H}$ and $\mathrm{D}$ plasma. Mechanical polishing, only conducted for SS5, does not affect $\sigma$, which suggests that surface impurities affecting $\sigma$ are not removed by the polishing.

In the case of the $\mathrm{W}$ samples, the evolutions of the deuterium density can be well reproduced by calculation, as shown in Fig. 5. The values of $\sigma$ for W3-2 and W4-2 are larger than those for the other runs of W. This would be due to surface impurities in the sample. $\sigma$ cannot be estimated during run 1 of the SS1 to SS5 and W1 to W2 samples, as denoted by N in Table 1, because the deuterium density rapidly increases within a very short time. This behavior is also expected for the hydrogen density on the SS6 sample. As the samples have not been chemically cleaned, impurities are present on the surface before exposure to plasma. It is understood that these impurities act as additional adsorption sites for hydrogen and are gradually removed by plasma exposure. As hydrogen would be weakly chemisorbed by these impurities, $\sigma$ on an impurity-covered surface is large. This is the case for the first exposure of D plasma to the W3 and W4 samples and therefore the data of W3-2 and W4-2 should be omitted from this study.

As there are no significant differences in $\sigma$ between $\mathrm{H}$ and $\mathrm{D}$ plasma exposure, the total averaged values of $\sigma$ for SS and W are $6.9 \pm 2.3 \times 10^{-23} \mathrm{~m}^{2}$ and $4.6 \pm 1.0 \times 10^{-23} \mathrm{~m}^{2}$, respectively. It is expected that $\sigma$ for $\mathrm{W}$ is smaller than that for SS because tungsten can chemisorb hydrogen atoms strongly [18]. The above result indicates, however, the difference is not clear. More precise measurements are needed for future works.

The impact desorption cross section of the D atom on the SS surface by $300-1500 \mathrm{keV}$ $\mathrm{H}^{+}$bombardment is very large, in the order of $10^{-20} \mathrm{~m}^{2}$, and is explained by a binary collision model [6]. This suggests that a hydrogen atom can be desorbed by the replacement process when it receives sufficient kinetic energy to escape from the surface. Impact desorption or replacement did not, and was not expected to, occur in the present work because a kinetic energy of $1 \mathrm{eV}$ is too low to remove a hydrogen atom, which is strongly chemisorbed on the surface [18]. Therefore recombinative desorption is considered to be the main mechanism of hydrogen 
desorption and $\sigma$ was found to be nearly equal to $\sigma_{\mathrm{d}}$ in the present work. As a hydrogen atom is chemically active, this process can occur even at a low kinetic energy. This is supported by the fact that hydrogen adsorbed on the surface is more effectively removed by hydrogen plasma than by inert gas plasma $[3,9]$.

In this analysis, the surface coverage, $\theta_{0}$, at steady state is assumed to be unity. When $\sigma_{d}=6.9 \times 10^{-23} \mathrm{~m}^{2}$ and $S_{0}=5.0 \times 10^{19} \mathrm{~m}^{-2}$ are substituted into Eq. (5), $\theta_{0}$ is estimated to be 0.997 . Hence, this assumption is valid in the present work.

\section{Conclusion}

The desorption cross section of hydrogen adsorbed on a metal surface at room temperature has been experimentally evaluated by NRA under continuous plasma exposure. As the incident energy of hydrogen atoms from plasma is very low, it is understood that recombinative desorption dominates the desorption process and replacement can be neglected. There are no significant differences in the desorption cross section between $\mathrm{H}$ and $\mathrm{D}$, and its average value is $6.9 \pm 2.3 \times 10^{-23} \mathrm{~m}^{2}$ on the SS surface and $4.6 \pm 1.0 \times 10^{-23} \mathrm{~m}^{2}$ on the $\mathrm{W}$ surface. A very large value for the desorption cross section found during the early stages of plasma exposure can be explained by the fact that hydrogen atoms on an impurity-covered surface are easily desorbed. 


\section{References}

[1] J. Roth, E. Tsitrone, T. Loarer, V. Philipps, S. Brezinsek, A. Loarte et al., Plasma Phys. Control. Fusion, 50 (2008) 103001 (20pp).

[2] L.D. Horton, P. Andrew, G. Bracco, S. Conroy, S. Corti, J. Ehrenberg et al., J. Nucl. Mater., 196-198 (1992) 139-142.

[3] H. Nakamura, S. Higashijima, K. Isobe, A. Kaminaga, T. Horikawa, H. Kubo et al., Fusion Eng. Design, 70 (2004) 163-173.

[4] T. Tanabe, K. Sugiyama, T. Shibahara, Y. Hirohata, M. Yoshida, K. Masaki et al., J. Nucl. Mater., 390-391 (2009) 705-708.

[5] R.S. Blewer, R. Behrisch, B.M.U. Scherzer, R. Schlz, J. Nucl. Mater., $76 \& 77$ (1978) 305312.

[6] R. Bastasz, L.G. Haggmark, J. Nucl. Mater., 103\&104 (1981) 499-502.

[7] R. Bastasz, L.G. Haggmark, J. Nucl. Mater., $111 \& 112$ (1982) 805-808.

[8] J. Roth, T. Schwarz-Selinger, V.Kh. Alimov, E. Markina, J. Nucl. Mater., 432 (2013) 341347.

[9] T. Hino, Y. Yamauchi, Y. Kimura, N. Nishikawa, Y. Ueda, Fusion Eng. Design, 87 (2012) 876-879.

[10] V.Kh. Alimov, B. Tyburska-Püschel, M.H.J’t Hoen, J. Roth, Y. Hatano, K. Isobe et al., Phys. Scr., T145 (2011) 014037 (5pp).

[11] I. Takagi, R. Imade, Y. Ikegami, M. Akiyoshi, K. Moritani, T. Sasaki et al., J. Nucl. Mater., 417 (2011) 564-567.

[12] M. Akiyoshi, H. Sakamoto, R. Haraguchi, K. Moritani, I. Takagi, H. Moriyama, Nucl. Instr. Meth. Phys. Res., B232 (2005) 173-177.

[13] I. Takagi, R. Sugiura, K. Shirai, K. Higashi, Fusion Sci. Technol., 31 (2002) 902-906.

[14] I. Takagi, H. Hashimoto, H. Fujita, K. Higashi, Fusion Eng. Design, 41 (1998) 73-78.

[15] W. Eckstein, J.P. Biersack, Appl. Phys. A, 38 (1985) 123-129.

[16] I. Takagi, Y. Koga, H. Fujita, K. Higashi, J. Nucl. Mater., 290-293 (2001) 501-504.

[17] K. Akaishi, Y. Kubota, A. Uritani, Y. Hori, J. Nucl. Mater., 145-147 (1987) 275-279.

[18] P.J. Estrup, J. Anderson, J. Chem. Phys., 45 (1966) 2254-2260. 
Table 1 List of samples

\begin{tabular}{lllll}
\hline \multirow{2}{*}{ Sample } & \multicolumn{4}{c}{ Plasma/vacuum sequence and $\sigma\left(\times 10^{-23} \mathrm{~m}^{2}\right)$} \\
\cline { 2 - 5 } & Run 1 & Run 2 & Run 3 & Run 4 \\
\hline SS1 & D (N) & vacuum & - & - \\
\hline SS2 & D (N) & vacuum & H (9.5) & - \\
\hline SS3 & D (N) & H (4.9) & - & - \\
\hline SS4 & D (N) & H (8.2) & D (3.1) & - \\
\hline SS5 & D (N) & H (6.0) & D (4.4) & - \\
\hline SS6 & H & D (10) & H (7.3) & D (8.6) \\
\hline W1 & D (N) & vacuum & H (5.4) & - \\
\hline W2 & D (N) & H (4.5) & D (6.4) & - \\
\hline W3 & H & D (15) & H (4.3) & D (3.4) \\
\hline W4 & H & D (19) & H (3.2) & D (4.7)
\end{tabular}

Notes: SS and W; sample materials. $\mathrm{N}$; unable to estimate $\sigma$ 
Figure captions

Fig. 1. A typical depth profile of deuterium in SS304 under continuous exposure to deuterium plasma.

Fig. 2. Illustration of process concerned with hydrogen exchange on the surface. Open circles and closed circles represent a hydrogen atom on the surface and an incident hydrogen atom, respectively. The broken line represents hydrogen before the interaction. Each process is explained in the text.

Fig. 3. Time dependence of the deuterium density on SS and W samples after exposure to a vacuum.

Fig. 4. Time dependent change in the deuterium density of SS samples after switching from either $\mathrm{H}$ or $\mathrm{D}$ plasma to the other plasma.

Fig. 5. Time dependent change in the deuterium density of $\mathrm{W}$ samples after switching from either $\mathrm{H}$ or D plasma to the other plasma. 


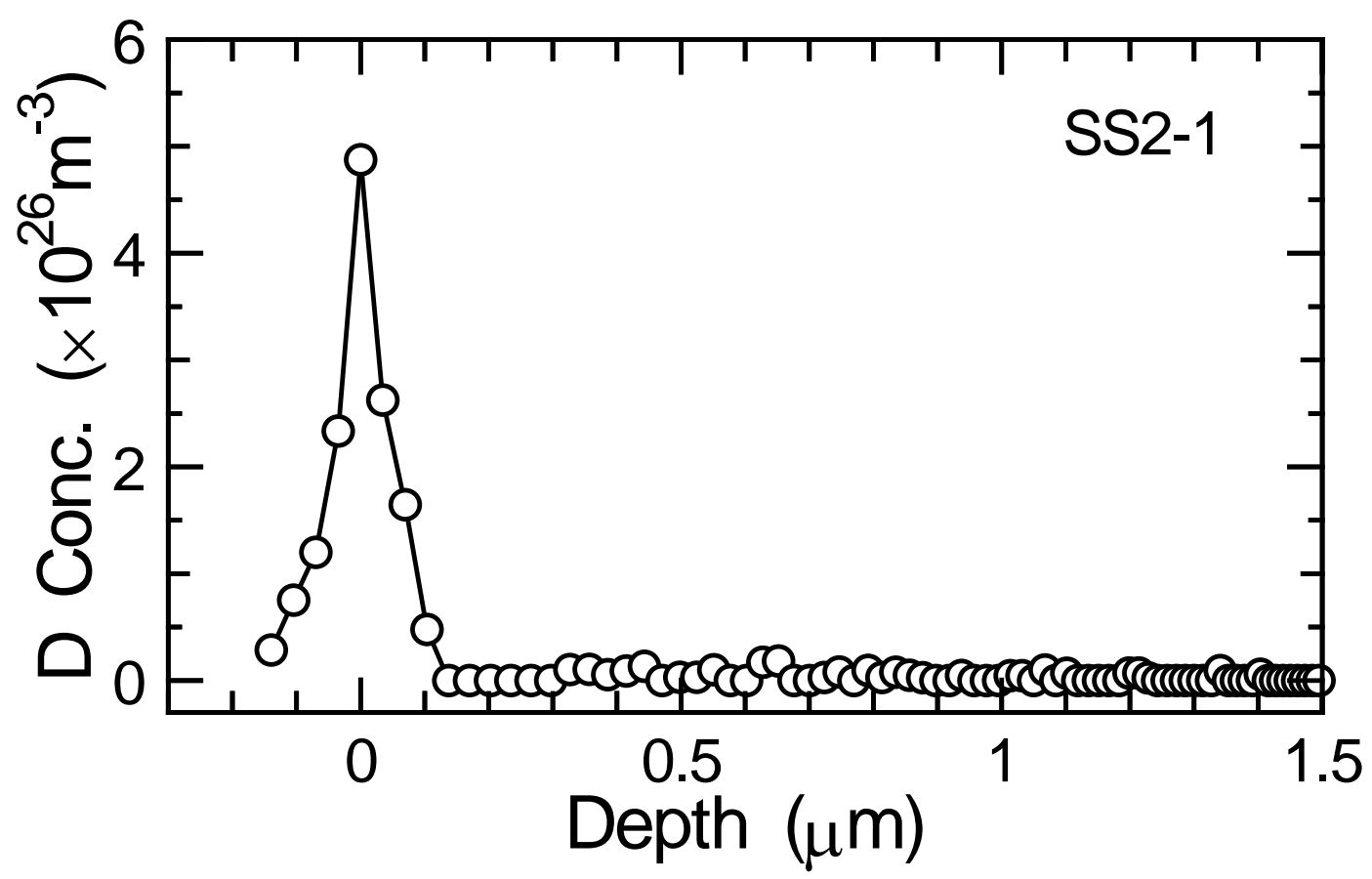

Fig. 1 

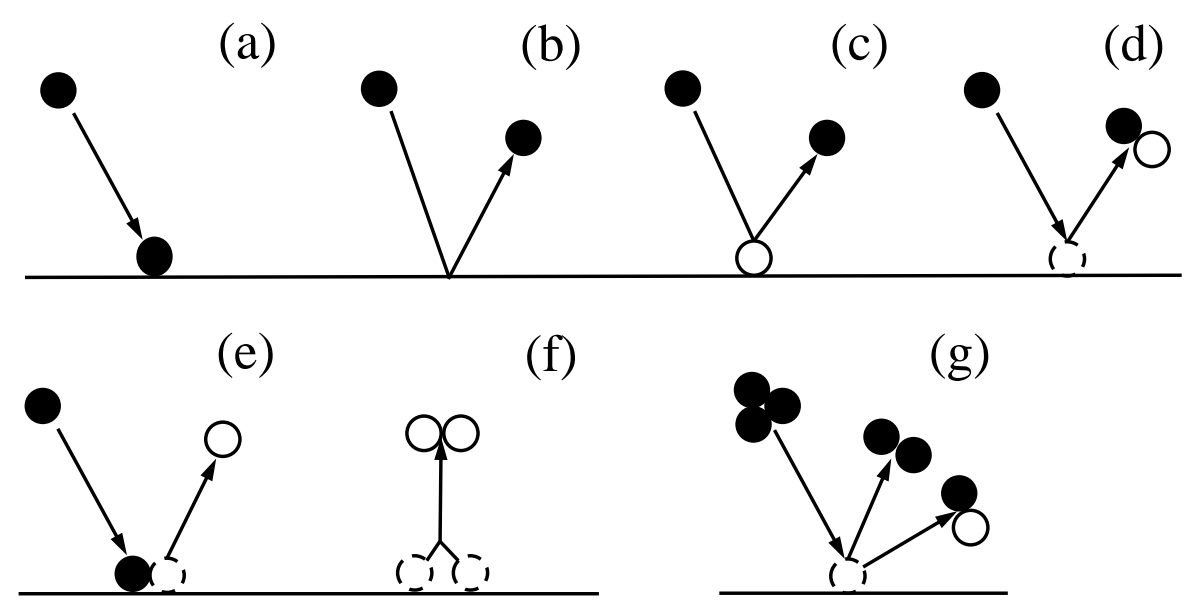

(g)

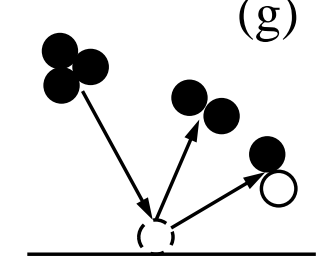

Fig. 2 


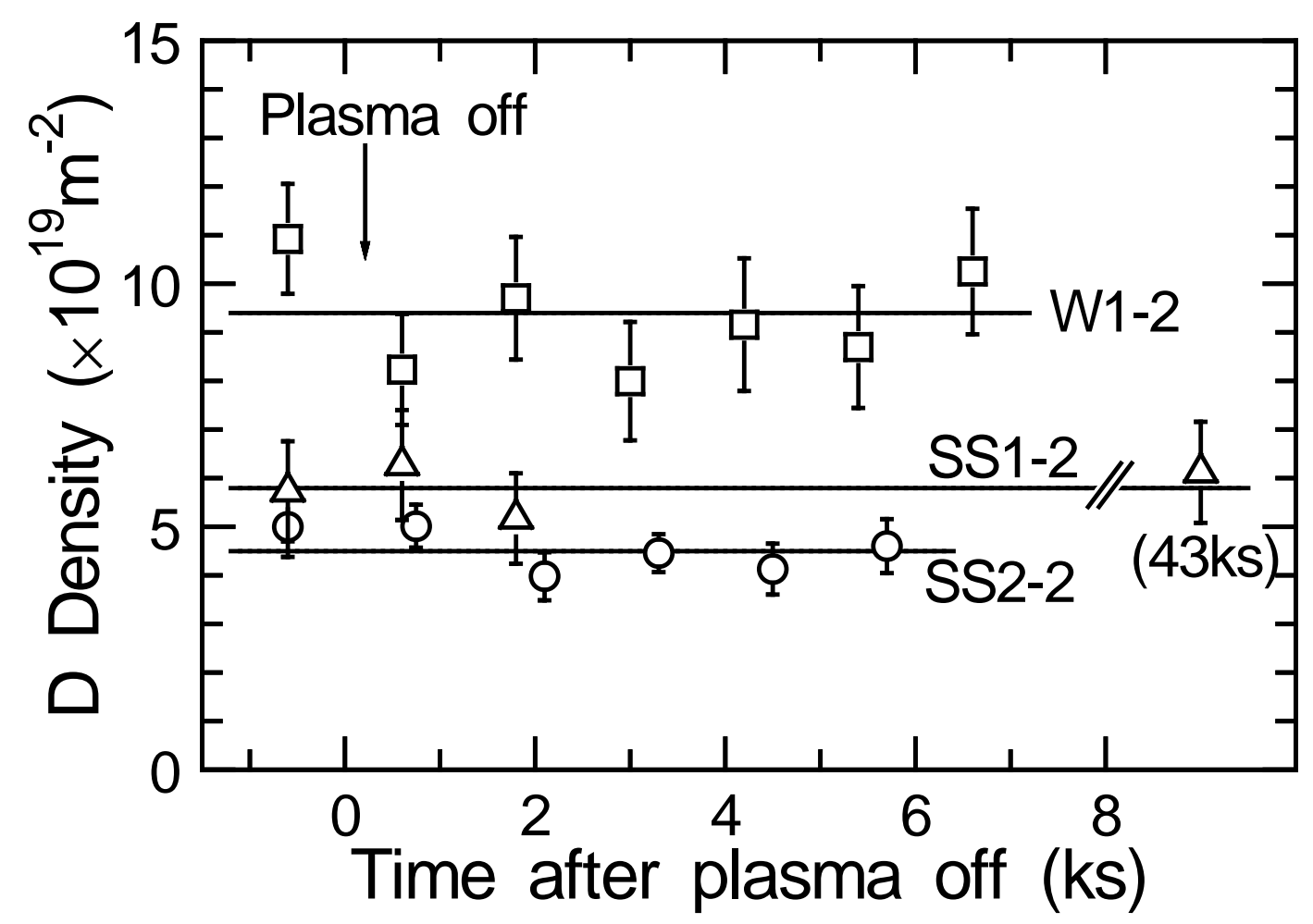

Fig. 3 


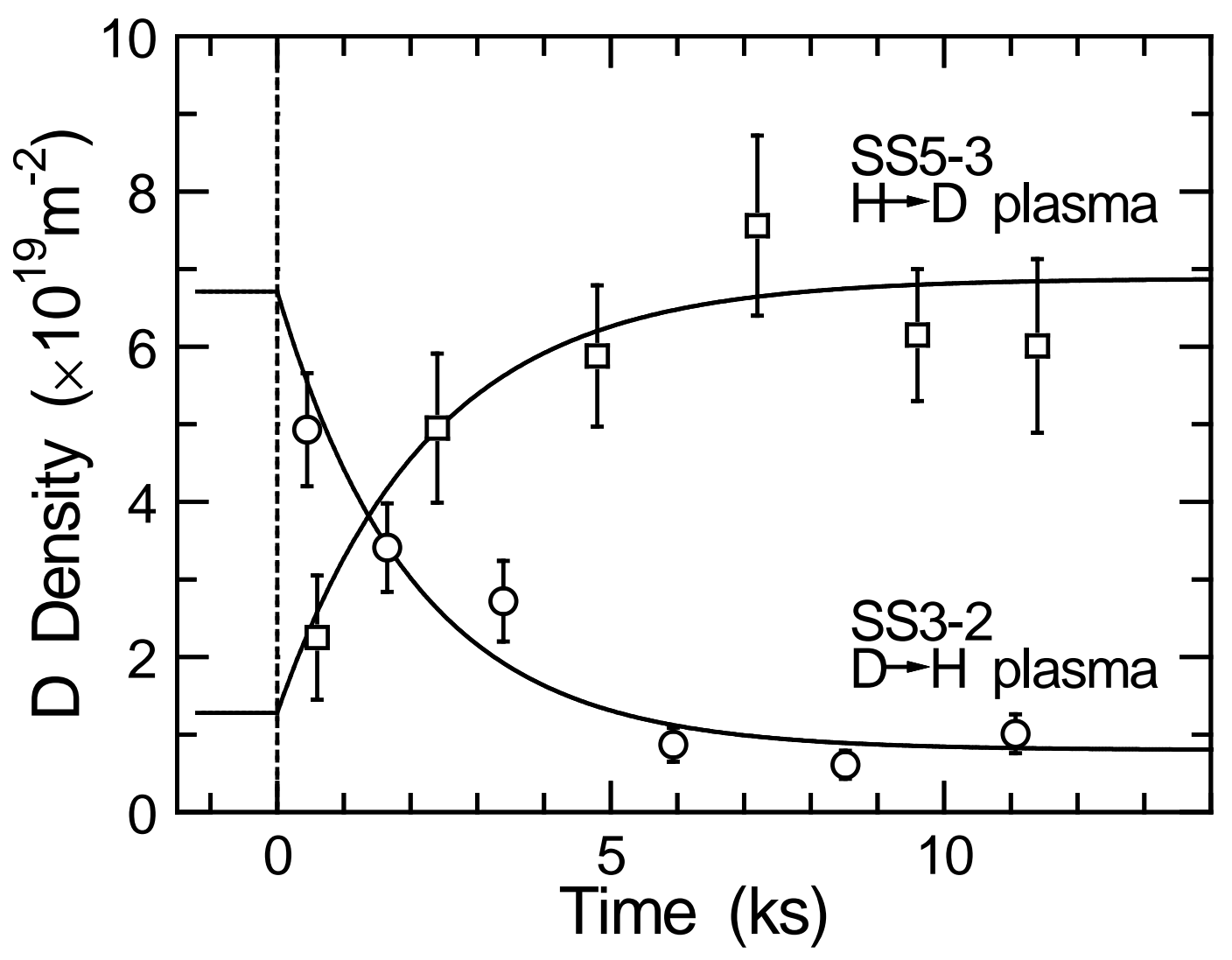

Fig. 4 


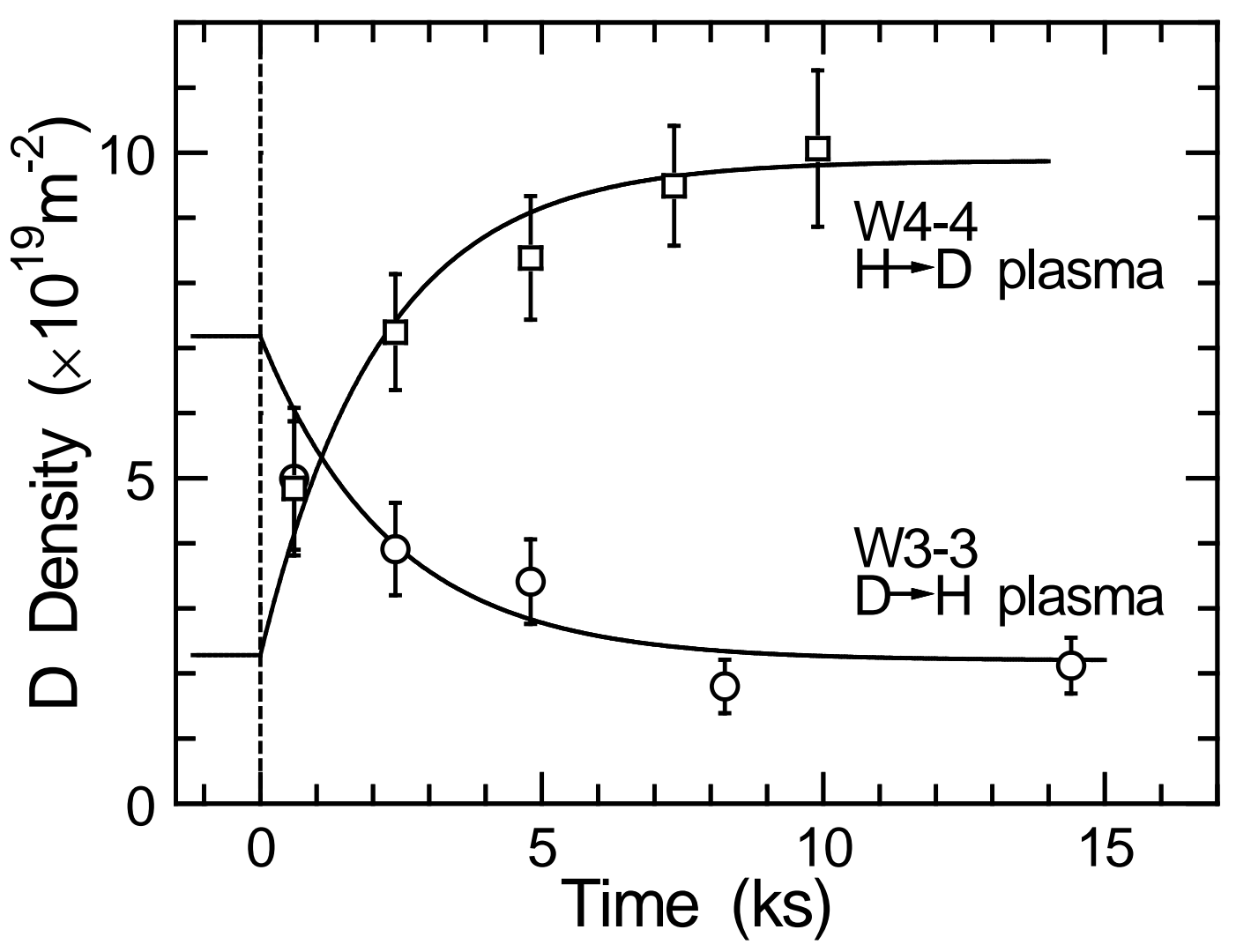

Fig. 5 